\title{
Optimising endocrine therapy in postmenopausal women with advanced breast cancer
}

\author{
Thomas Ho Lai Yau and Kwok-Leung Cheung \\ School of Medicine, University of Nottingham, Derby, UK \\ Correspondence should be addressed to K-L Cheung: kl.cheung@nottingham.ac.uk
}

\begin{abstract}
Hormone receptor-positive breast cancer is commonly treated with endocrine therapy (ET); however, over time, cancer cells can develop endocrine resistance. This review aims to document combination therapy and sequential therapy in the use of endocrine agents and targeted agents, by conducting two systematic searches using four databases: Cochrane Library, MEDLINE, EMBASE and Web of Science. A total of 26 studies that covered combination therapy were obtained and included for the review. Fourteen were phase III documenting combinations of mechanistic target of rapamycin (mTOR), phosphoinositide-3-kinase (PI3K), vascular endothelial growth factor receptor, human epidermal growth factor receptor 2 and cyclin-dependent kinase 4/6 (CDK4/6) inhibitors. The remaining studies were of phase II nature that reported combinations involving inhibitors in mTOR, endothelial growth factor receptor, CDK4/6 and TKI. Interesting findings in inhibitor combinations involving CDK4/6, mTOR and PI3K suggest clinical activity that can overcome endocrine resistance. On the other hand, there were 0 studies that covered sequential therapy. Overall findings showed that combination therapy improved treatment efficacy over monotherapy in postmenopausal patients with hormone receptor-positive advanced breast cancer. Inevitably, the benefits are accompanied with increased toxicity. To optimise ET, further research into combinations and effective patient selection will need to be defined. Additionally, this review warrants future studies to explore sequential therapy.
\end{abstract}

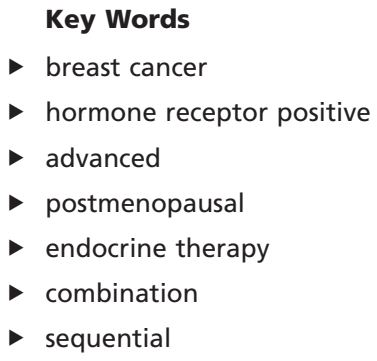

Endocrine-Related Cancer (2018) 25, 705-721

\section{Introduction}

Endocrine therapy (ET) is often used as first-line treatment in patients with hormone receptor-positive (HR+) breast cancer and preferred to chemotherapy when there are no signs of visceral crisis (Reinert \& Barrios 2015). In terms of efficacy, ET improves progression-free survival (PFS), time to progression (TTP), objective response rate (ORR) and clinical benefit response (CBR), while possessing a favourable toxicity profile when compared to chemotherapy. Although the therapeutic action of ET is slower than chemotherapy, the duration of response in ET is more sustainable with longer-term survival benefits (Cheung 2007). Recent data from the FALCON trial observed significant improvements in not just PFS and TTP but also overall survival (OS) for postmenopausal patients with endocrine-naïve, $\mathrm{HR}+$ locally advanced/ metastatic breast cancer (LABC/MBC) when treated 
with fulvestrant $500 \mathrm{mg}$, as opposed to anastrozole $1 \mathrm{mg}$ (Robertson et al. 2016). All HR+ breast cancers can be represented with the presence of oestrogen receptor (ER) and/or progesterone receptor (PR) (Cheung 2007). The American Soceity of Clinical Oncology/College of American Pathologists recommended HR+ tumours be defined as having at least $1 \%$ of tumour nuclei stained positively for either ER or PR on immunohistochemistry (Hammond et al. 2010). Unfortunately, patients with ER+ breast cancer are susceptible to risks of progressive disease (PD) or develop endocrine resistance (Dixon 2014). As a result, investigations in modalities of ET agents have been thorough and produced a wide range of ET options for patients to use.

A greater understanding in cancer biology has shown that ESR1 mutation is associated with mechanisms of endocrine resistance, especially to tamoxifen and fulvestrant (Jeselsohn et al. 2015). About 15-20\% of $\mathrm{ER}+\mathrm{LABC} / \mathrm{MBC}$ were shown to have ESR1 mutation, with increased frequencies detected in patients with multiple ET exposure. Research into biochemical pathways associated with proliferation has identified that cross-talk between signalling pathways can activate ERs, despite conventional ER pathways being blocked or inactivated (Pietras 2006, Dixon 2014). For instance, cross-talk between ER and specific pathways such as the phosphoinositide 3-kinase/v-akt murine thymoma viral oncogene/mammalian target of rapamycin (PI3K/AKT/ mTOR) can result in continued proliferation of the cancer cells and hence develop resistance to ET (Dixon 2014). Targeted therapy agents (TAs) are designed to interfere with specific targets that are involved with growth. Often TAs act on specific molecular targets to achieve blockade of cell proliferation and potential cross-talks between the ER-mediated pathway and other signalling pathways. Most TAs are categorised by their molecular target (Table 1). Accordingly, the concomitant use of TA with other cancer therapeutics can potentially further increase treatment efficacy and overcome endocrine resistance
(Pietras 2006). However, combination therapy is prone to a greater toxicity profile when compared to monotherapy. Hence, an alternative would be the sequential application of ET and TA, which is expected to lessen the toxicity profile of these regimens. In sequential therapy, the patient will be exposed to only one toxicity profile at once rather than two during combination therapy. From Fig. 1, it was of interest if sequential application of an ET agent and TA will produce similar efficacy when compared to combination therapy. Another interesting comparison of these treatments would be to compare the results of different sequencing pattern in sequential ET (in this case treatment B and C). Henceforth, this was the definition of combination therapy and sequential therapy in this review.

An ever-growing arsenal of anticancer agents requires knowledge in optimal application for clinicians and patients to make informed decisions regarding therapeutic strategies. The aim was to assimilate methodologies and conclusions of randomised control trials (RCTs) investigating the benefits/limitations of combination and sequential therapy of ET/TA.

\section{Methods}

This systematic review was conducted by electronic searches to include relevant phase II/III RCTs that have reviewed the application of ET and TA in combination therapy or sequential therapy. Relevant literatures were screened for their title, followed by evaluation of abstracts befitting the selection criteria. Lastly, availability of full articles and abstracts in eligible literature were reviewed. Two separate searches were performed in parallel to accommodate the aims of the review.

A comprehensive search was performed with multiple databases: Medline, EMBASE, Cochrane Library and Web of Science. Both searches included 'endocrine therapy', 'hormone', 'advanced breast cancer', 'metastatic' and 'postmenopausal'. Additional search terms: 'combination',

Table 1 Some targeted therapy agents that have been used in treating breast cancer in combination with other forms of cancer treatment.

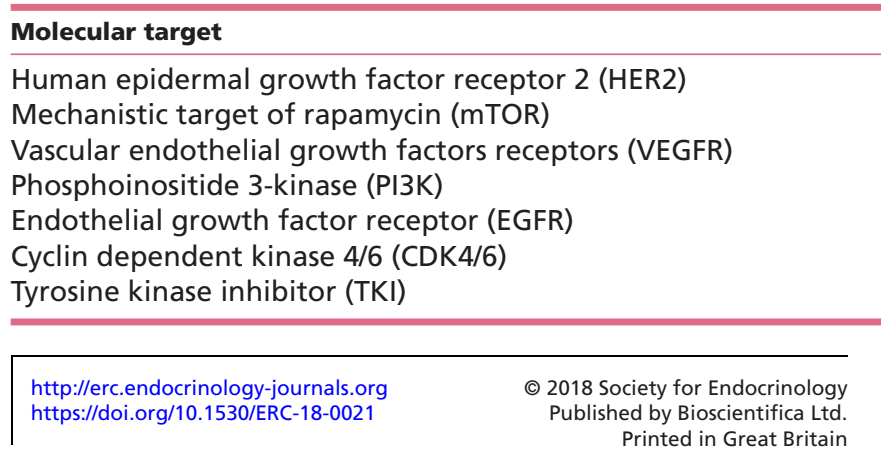

\begin{tabular}{l} 
Action \\
\hline Inhibit \\
Inhibit \\
Inhibit \\
Inhibit \\
Inhibit \\
Inhibit \\
Inhibit
\end{tabular}

\begin{tabular}{l}
\hline Example(s) \\
\hline Trastuzumab, lapatinib \\
Everolimus, temsirolimus \\
Bevacizumab \\
Buparlisib \\
Gefitnib, sapatinib \\
Palbociclib, ribociclib \\
Dovitinib
\end{tabular}


Combination therapy vs Sequential therapy

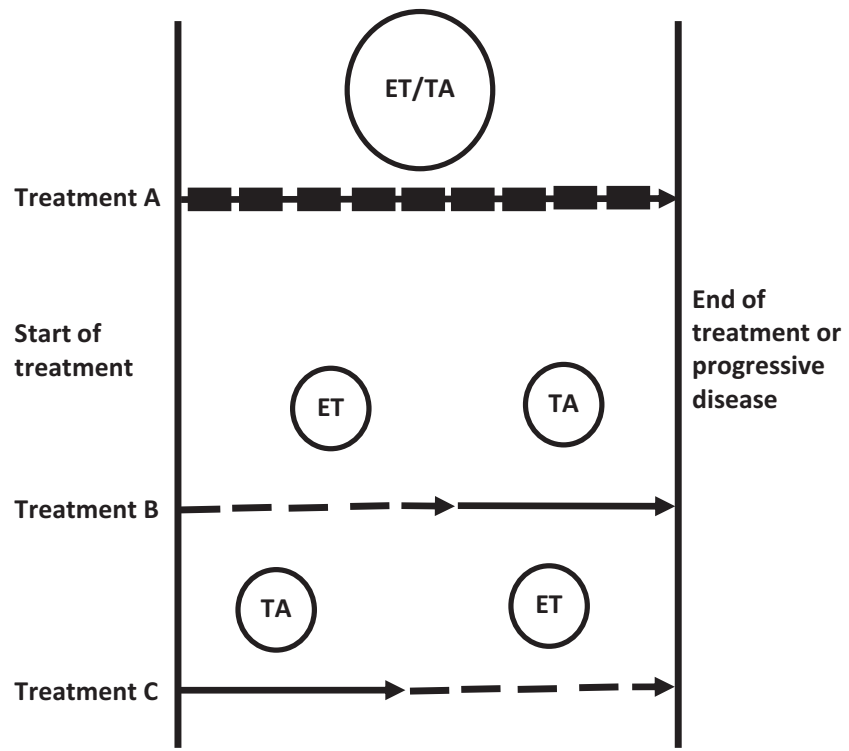

Figure 1

A hypothetical comparison of combination therapy (treatment $A$ ) and sequential therapy (treatment $B$ and C). ET, endocrine therapy agent; TA, targeted agent; ET/TA, combination of endocrine therapy agent and targeted agent; Blocked arrow, duration of effective treatment from ET/TA; Dashed arrow, duration of effective treatment from ET; Straight arrow, duration of effective treatment from TA.

'plus', 'add' and 'together' were incorporated into the search for combination therapy. Whereas, search terms: 'sequential', 'switch', 'concurrent', and 'concomitant' were included for the sequential therapy search. Crossreferencing of relevant literature was also conducted to expand the literature search. Conference abstracts were also considered for screening, to include on-going studies for review. The search was limited to English language and RCTs that investigated combinations or sequential applications of ET and TA in postmenopausal patients with HR+ advanced/MBC in phase II/III. The search was carried out from 1998 onwards, because trastuzumab was approved by the Food and Drug Administration on this year (Roche \& Ingle 1999). The Critical Appraisal Skill Programme (CASP) RCT checklist was used for critical appraisal of founded studies.

\section{Inclusion criteria}

- ET combination with TA

- Sequential use of ET with TA

- Primary interest of ET agents includes:

o Selective oestrogen receptor modulators (SERMs): tamoxifen o Steroidal third-generation aromatase inhibitors (AIs): exemestane

o Non-steroidal third-generation AIs: anastrozole or letrozole

o Selective oestrogen receptor downregulators (SERDs): fulvestrant

- Study title must be a RCT that report any of the following molecular TA with ET:

o HER2 inhibitors

o mTOR inhibitors

o CDK4/6 inhibitors

o VEGFR inhibitors

o EGFR inhibitors

o PI3K inhibitors

o TKIs

- Study must offer full text or abstract that provide details in:

o Background/Introduction

o Methods

o Results

o Discussion/Conclusion

- HR+ breast cancer may include:

o ER+, PR+, HER2+

o ER+, PR+, HER2-

o ER+, PR-, HER2-

o ER+, PR-, HER2+

o ER-, PR+, HER2+

o ER-, PR+, HER2-

- Study must recruit postmenopausal patients or in addition to premenopausal patients

- Prior chemotherapy was acceptable in abstract screening of RCTs

\section{Exclusion criteria}

- Keywords 'chemotherapy' or 'radiotherapy' stated in title or in combination with ET

- Combination of ET agents (SERDs, AIs, SERMs)

- 'Premenopausal' or 'early breast cancer' stated in title

- Study solely on premenopausal patients

- Non-human studies

- Neo-adjuvant studies

\section{Primary outcome}

The primary objective was to evaluate the effectiveness of combination therapy and sequential therapy in optimising ET. The optimisation of ET will be measured by observed improvements in PFS, ORR, TTP, CBR and OS. Remarks of overcoming endocrine resistant will also be considered. 


\section{Secondary outcome}

The benefits and limitations of combination therapy and sequential therapy were evaluated. Parameters included quality of life (QoL), toxicity and cost-effectiveness will also be considered.

It was hypothesised that combination therapy was a more suitable option to optimising ET when compared to sequential therapy in terms of improving treatment efficacy and overcoming endocrine resistance.

\section{Results}

\section{Combination therapy search}

From Fig. 2, an initial detection of 2866 articles from the 4 databases. A final total of 26 studies were achieved, after removal of duplicates, title and abstract screening according to the inclusion and exclusion criteria stated in methods.

From Table 2, there are 9 studies addressing ET/mTOR, 3 ET and CDK4/6, 1 study addressing ET/PI3K, 3 studies addressing ET/HER2, 2 studies addressing ET/VEGFR, 5 studies addressing ET/EGFR and 3 studies addressing ET/TKI combinations. 2 studies had CBR as their primary endpoint and the rest of the studies had PFS.

\section{ET combinations with mTOR inhibitors (phase III/II)}

The combination of exemestane and everolimus was well documented in the international, phase 3 , multicentre, randomised, double-blind, placebo-controlled trial: BOLERO-2 (Baselga et al. 2012, Piccart et al. 2012, Burris et al. 2013a,b, Yardley et al. 2013). The targeted population consisted of postmenopausal women with

\section{Studies addressing combination ET with TA}

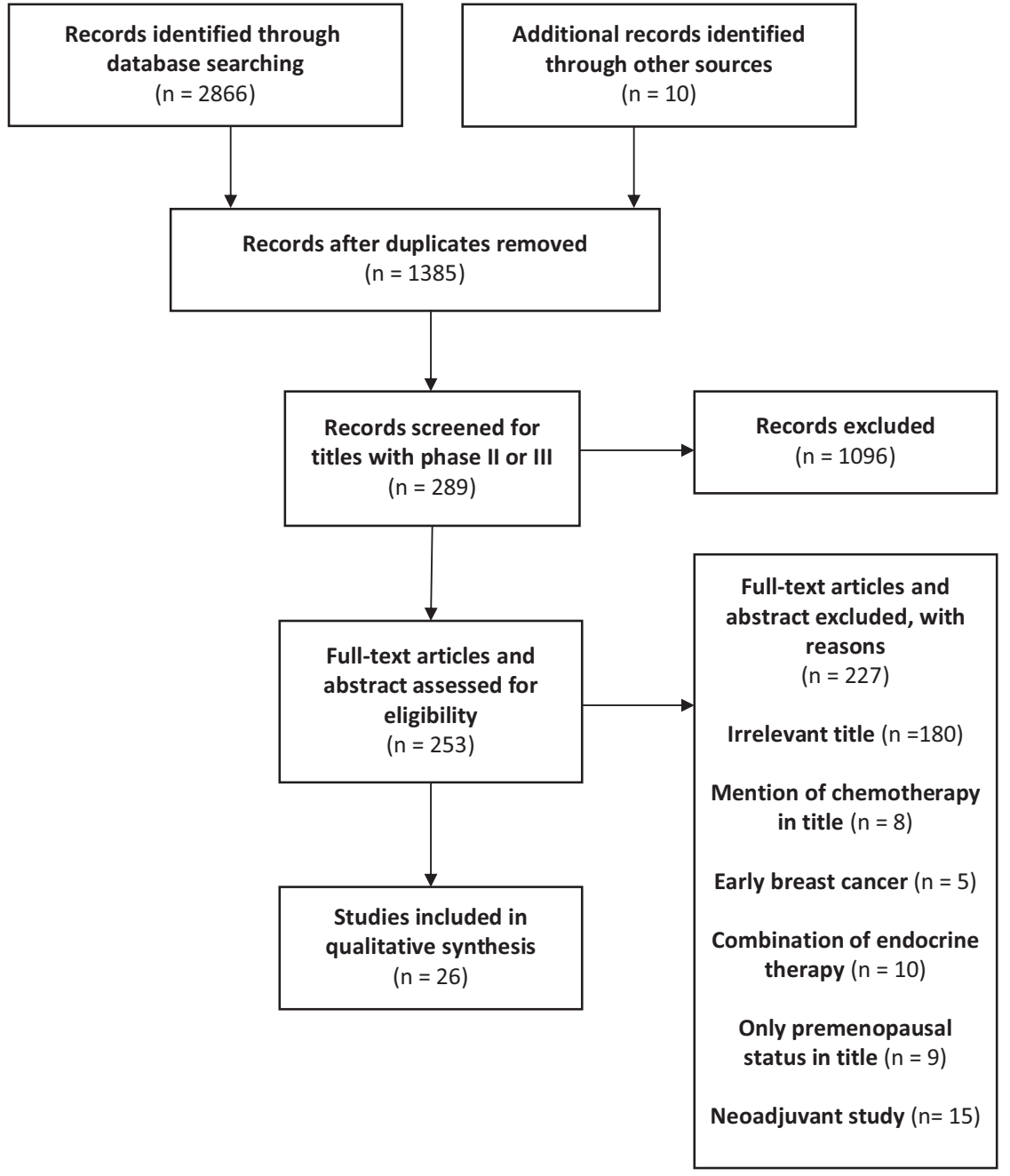

\section{Figure 2}

A flow diagram displaying the study selection process that addressed for combinations of ET with targeted agents. Adapted, under the terms of the Creative Commons Attribution License, from Moher D, Liberati A, Tetzlaff J, Altman DG, The PRISMA Group (2009) Preferred Reporting Items for Systematic Reviews and Meta-Analyses: The PRISMA Statement. PLoS Medicine 6(7): e1000097. 
Table 2 Summary of included phase II/III studies that address combination of ET and TA (mTOR inhibitors; CDK4/6 inhibitors; PI3K inhibitor; HER2 inhibitors; VEGFR inhibitor; EGFR inhibitor and TKI).

Authors
Bachelot et al. (2012)
Baselga et al. (2012),
Piccart et al. (2012),
Burris et al. (2013a,b),
Yardley et al. (2013)
Carpenter et al. (2005),
Wolff et al. (2013)
Kornblum et al. (2017)
Baselga et al. (2016)
Burstein et al. (2014)
Johnston et al. (2009)
Kaufmann et al. (2009)
Cristofanilli et al. (2016)
Finn et al. (2016a,b)
Martin et al. (2015),
Dickler et al. (2016)
Martin et al. (2015)
Osborne et al. (2011)
Valero et al. (2009)
Cristofanilli et al. (2010),
Carlson et al. (2012),
Tryfonidis et al. (2016)
Carlson et al. (2012)
Wright et al. (2011)
Musolino et al. (2017)
Johnston et al. (2016)
Thion

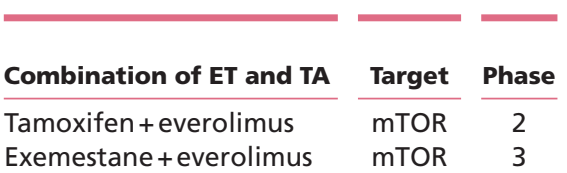

\begin{tabular}{ccc}
$\begin{array}{c}\text { Number of } \\
\text { studies }\end{array}$ & $\begin{array}{c}\text { Number of } \\
\text { patients }\end{array}$ \\
\cline { 1 - 1 } 1 & & 111 \\
5 & & 722 \\
\end{tabular}

\begin{tabular}{c}
$\begin{array}{c}\text { Primary } \\
\text { endpoint }\end{array}$ \\
\hline CBR \\
PFS
\end{tabular}

Abstract or Full text

Exemestane+everolimus

mTOR

Letrozole +temsirolimus

Fulvestrant + everolimus Fulvestrant + buparlisib

Fulvestrant + lapatinib Letrozole + lapatinib Anastrozole + trastuzumab

Fulvestrant + palbociclib Letrozole + palbociclib

Letrozole + bevacizumab

$\begin{array}{cr}\text { mTOR } & 3,2 \\ \text { mTOR } & 2 \\ \text { PI3K } & 3 \\ \text { HER2 } & 3 \\ \text { HER2 } & 3 \\ \text { HER2 } & 3 \\ \text { CDK4/6 } & 3 \\ \text { CDK4/6 } & 3,2 \\ \text { VEGFR } & 3 \\ & \\ \text { VEGFR } & 3 \\ \text { EGFR } & 2 \\ \text { EGFR } & 2\end{array}$

Fulvestrant + bevacizumab Tamoxifen + gefitinib Anastrozole + gefitinib

Fulvestrant + gefitinib

Fulvestrant + dasatinib

Fulvestrant + dovitinib

Anastrozole +AZD8931

\begin{tabular}{l} 
EGFR \\
TKI \\
TKI \\
TKI \\
\hline
\end{tabular}

\begin{tabular}{ll}
2 & 1 \\
2 & 1 \\
2 & 1 \\
2 & 1 \\
\hline
\end{tabular}

$\begin{array}{r}* * 141 \\ 99 \\ 97,71 \\ 359 \\ \hline\end{array}$

CBR
PFS
PFS
PFS

Full text

Full text, Full text, Abstract, Abstract, Full text

Data with * and ** represent data from the same study

HR+, HER2- locally ABC or MBC whom experienced PD from letrozole or anastrozole. Eligible patients were randomised in a blind manner at a 2:1 ratio for the experimental arm $(25 \mathrm{mg} /$ day exemestane and $10 \mathrm{mg}$ /day oral everolimus) or matching placebo. The investigation in BOLERO-2 showed significant improvements in PFS and other efficacy parameters (Table 3). These improvements in efficacy were also maintained in patients with visceral disease, elderly and of Asian ethnicity. Thus, the everolimus/exemestane combination represents an improvement in managing a wider population of postmenopausal women with HR+, HER2-ABC. Furthermore, BOLERO-2 is the only study that reported QoL. Burris et al. reported similar baseline global health status score in treatment and placebo regimen (64.7 vs 65.3) (Burris et al. 2013b). The similar outcome of QoL further supports the use of everolimus with ET.

Despite BOLERO-2 advocated the benefits of using mTOR inhibitor, contrasting finding in PFS was observed in the HORIZON study (Wolff et al. 2013). This study investigated the use of letrozole in combination with oral mTOR inhibitor temsirolimus in the phase III setting, following positive findings of improvement in PFS and CBR from previous phase II trial (Carpenter et al. 2005). This combination failed to improve PFS (8.9 vs 9.0 months), ORR ( $27 \%$ vs $27 \%$ ) and OS. Moreover, a raised toxicity profile in the combination arm resulted in more grade $3 / 4$ adverse events (AEs) (37\% vs $24 \%$ ). However, it was speculated that the contrasting findings in both trials were due to key differences in eligible patient characteristics (Wolff et al. 2013). For instance, HORIZON excluded patients with prior AI exposure within 12 months, whereas eligible patients in BOLERO-2 required progression from a non-steroidal AI during or within 12 months. This speculation highlights the significance of patient selection to determining the success of the treatment regimen. Interestingly, it was noted in the HORIZON study observed an improved PFS (9.0 vs 5.6 months) limited to patients aged $\leq 65$ years treated with the combination letrozole/temsirolimus rather than in patients aged $\geq 65$ (8.5 vs 10.1 months). This finding suggests that temsirolimus activity may favour the younger population over the older population (Wolff et al. 2013). Again, this proposal accentuates the importance of patient selection for treatment success. 

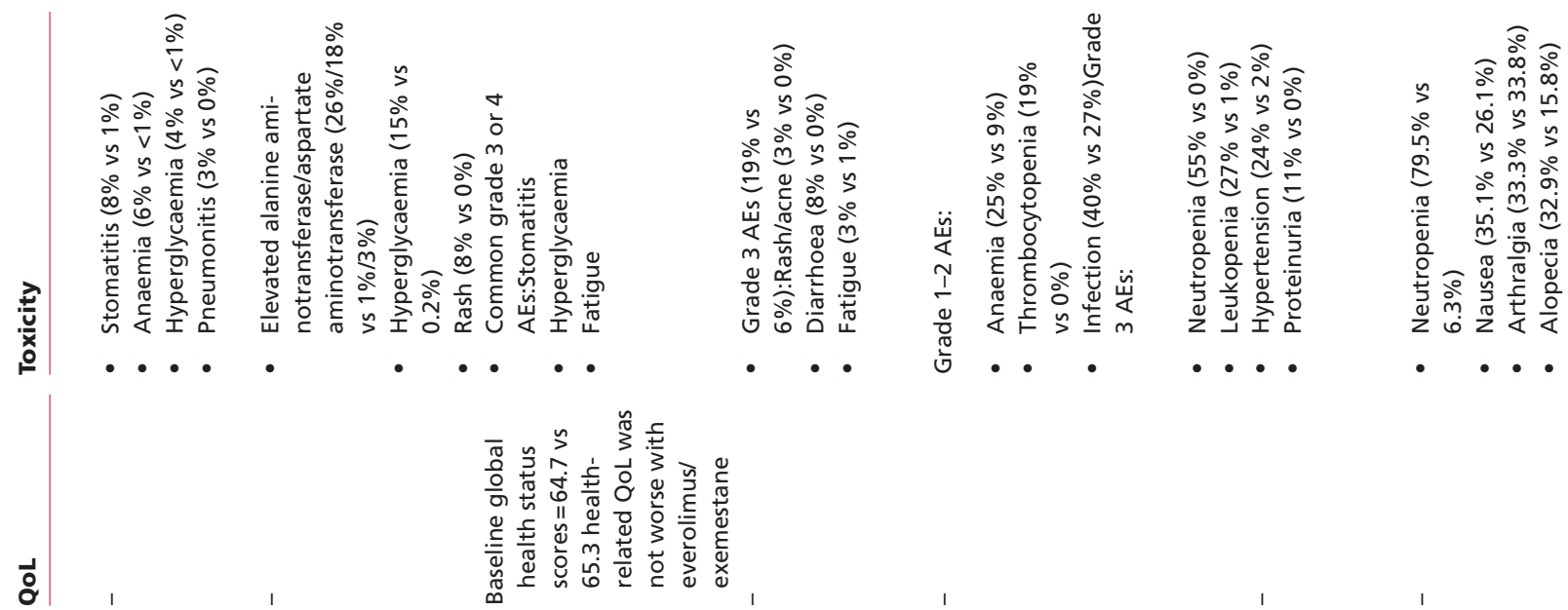

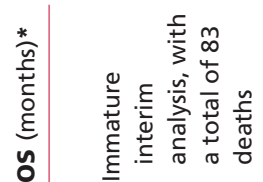

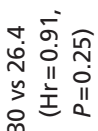
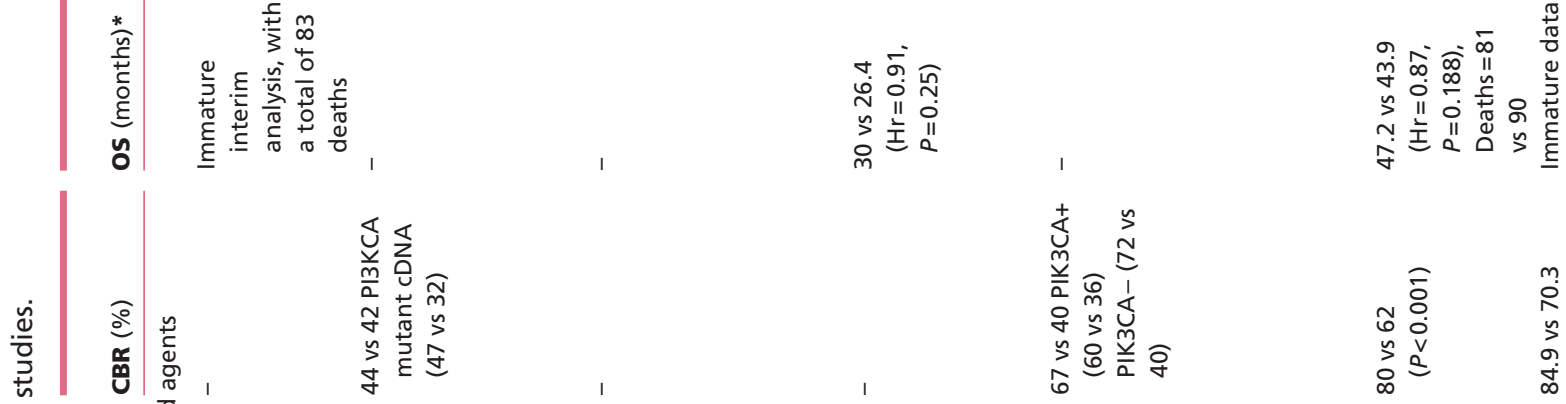

$\begin{array}{rl} & \widehat{\bar{o}} \\ \therefore & 8 \\ 5 & 0 \\ 0 & v \\ 0 & =\end{array}$
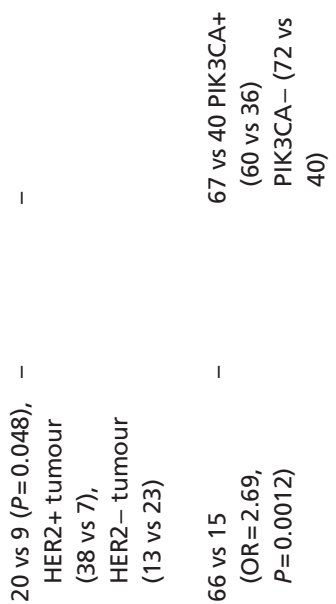

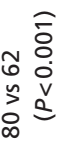

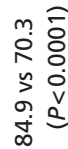

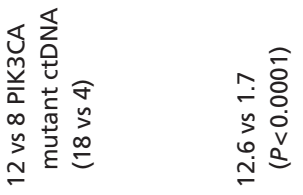

0
0
5

0
0

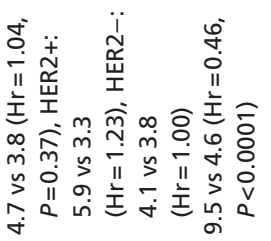

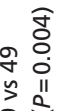

寅商

वृ
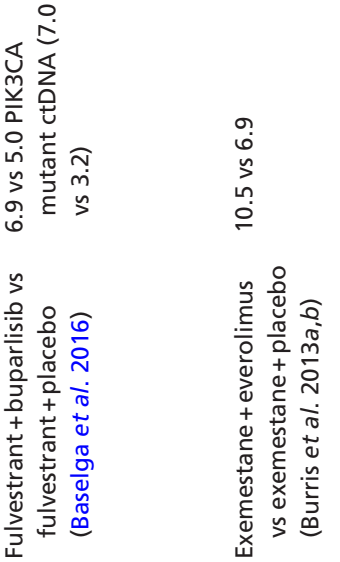
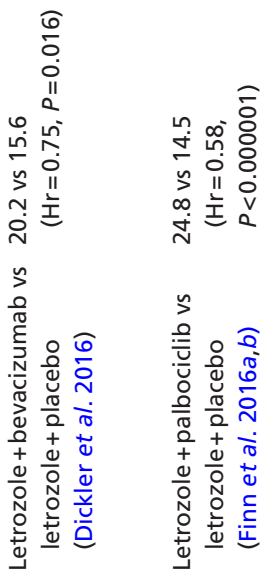


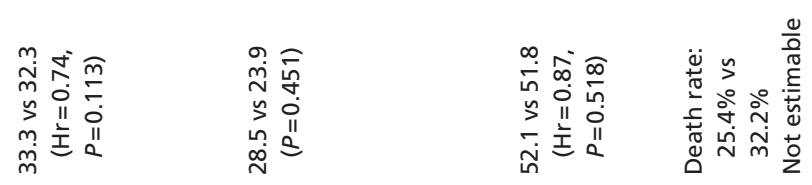

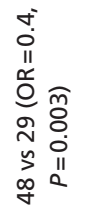


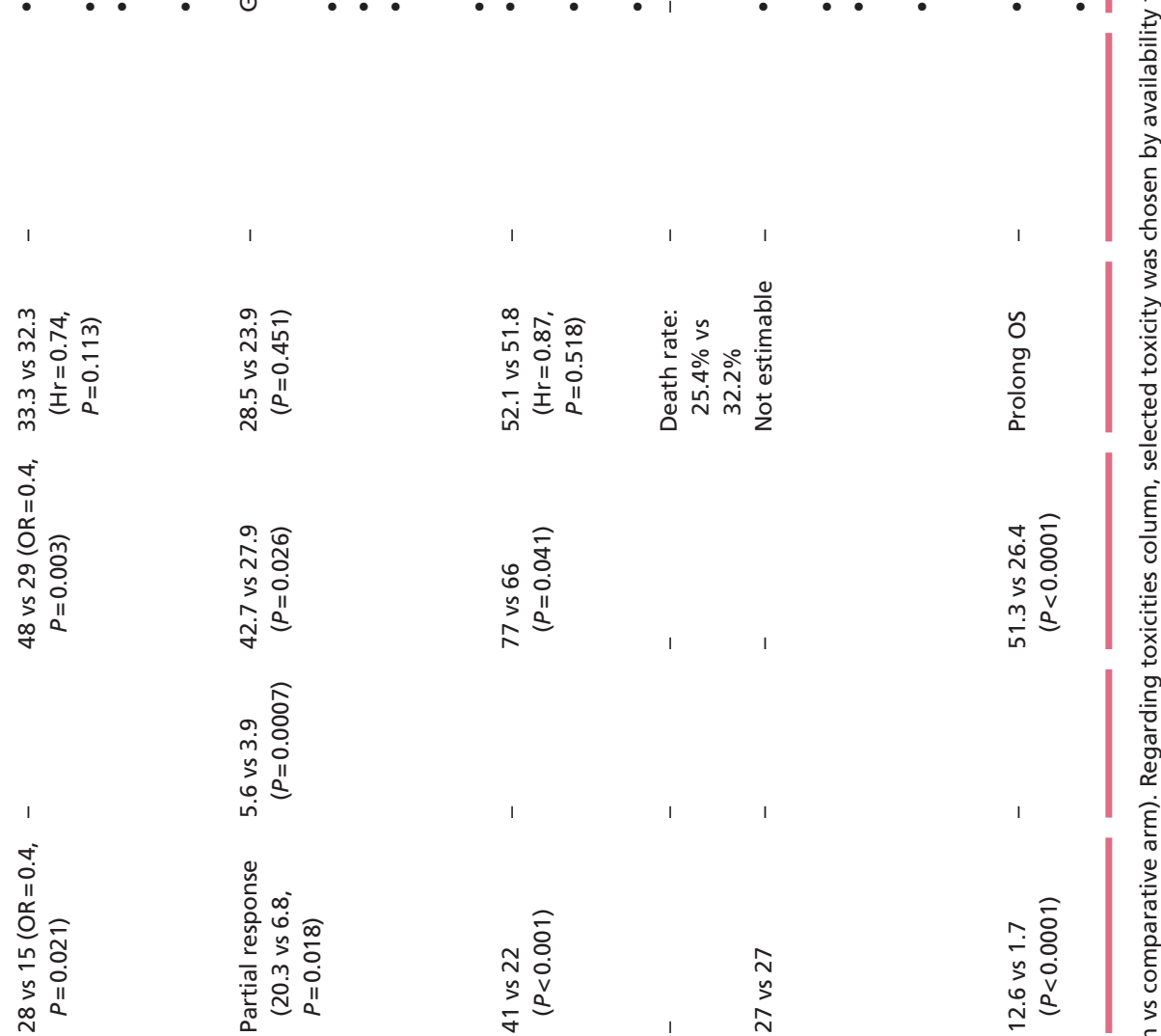

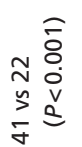
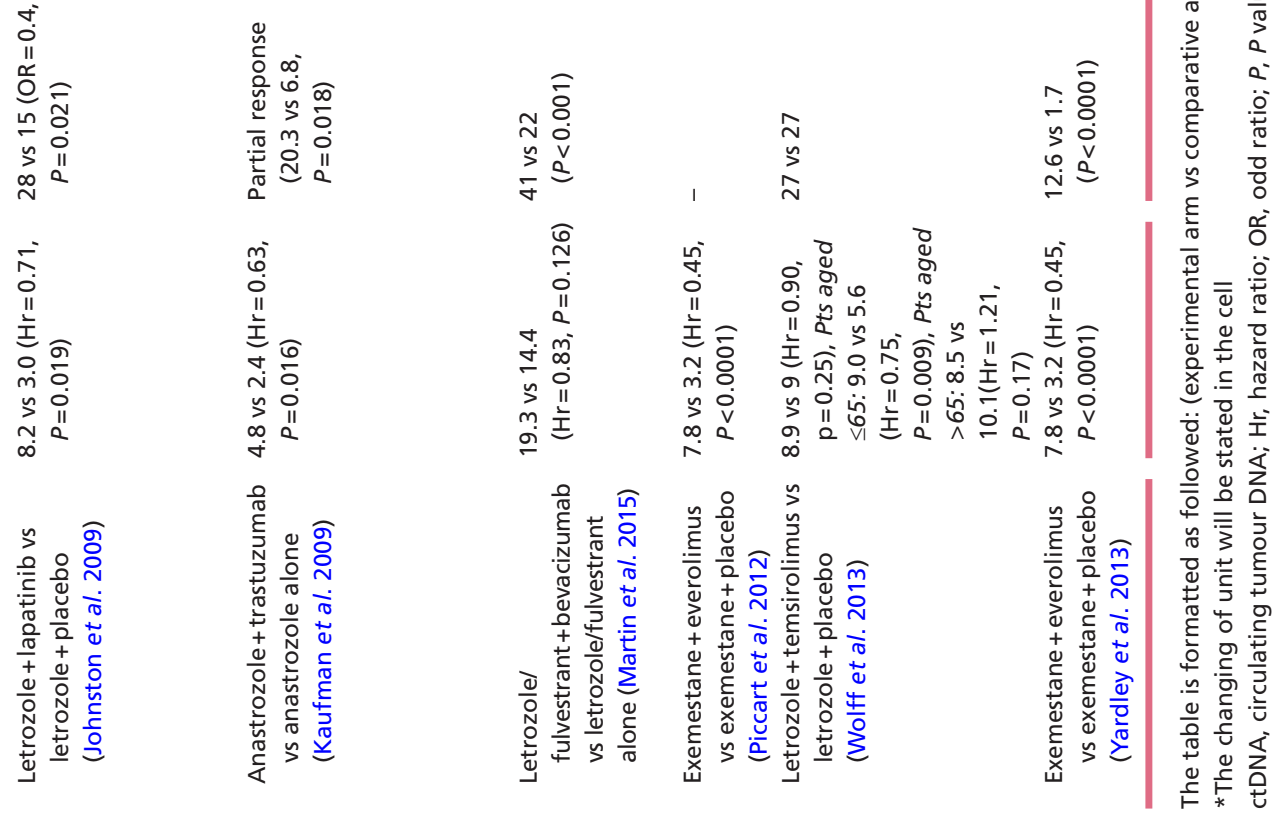
From the open-labelled RCT (TAMRAD) that investigated the tamoxifen/everolimus combination. An interesting finding in CBR suggested possible reversal of ET resistance and subsequent improvements. Overall, CBR at 6 months was $61 \%$ vs $42 \%$. Moreover, improvements in CBR were consistent in patients with secondary resistance (74\% vs $48 \%$ ) and in patients with primary resistance (46\% vs $38 \%$ ). Similar findings in TTP (14.8 vs 5.5 months) was more prominent in patients with secondary resistance as opposed to those with primary resistance (5.4 vs 3.8 months) (Bachelot et al. 2012). Therefore, this combination may benefit patients with AI-resistant MBC. However, this trial was relatively small with a total of 111 patients and may be prone to bias. Small imbalances between groups' performance status were notable (Bachelot et al. 2012). Hence, this study was confirmed only for hypothesis generating and warrant further study into this area (Bachelot et al. 2012). An alternate combination of fulvestrant and everolimus has shown positive improvements in PFS (10.4 vs 5.1 months) against AI-resistant MBC in the phase II settings (Kornblum et al. 2017). Once again, this highlights the potential of combination therapy in overcoming resistance.

\section{ET combinations with CDK4/6 inhibitor (phase III/II)}

Positive results were observed when novel CDK4/6 inhibitor palbociclib was added to ET. From Table 3, PALOMA-2 (letrozole/palbociclib) and PALOMA-3 (fulvestrant/palbociclib) have shown improvements in efficacy parameters. In both PALOMA-2 and PALOMA-3, significant improvements in PFS, ORR and CBR were reported. In terms of toxicity, neutropenia (79.5\% vs $6.3 \%$ ) was evident when palbociclib was added. Nonetheless, PALOMA-2 confirmed the significant clinical benefits and safety of using palbociclib/letrozole to treat postmenopausal patients whom had no prior systemic therapy for their ER+, HER2- ABC (Finn et al. $2016 a, b)$.

From PALOMA-3, patients with HR+, HER2- MBC were randomised in a double-blind manner to fulvestrant (500 mg, intramuscular injections on days 1 and 15 of cycle one and then on day 1 of each 28-day cycle) and palbociclib or placebo $(125 \mathrm{mg} /$ day oral for 3 weeks, followed by 1 week off in a 28-day cycle). Although this trial recruited both premenopausal and postmenopausal women, premenopausal women were treated with goserelin (LHRH agonist) to induce postmenopausal status.
Significant improvements in PFS (9.5 vs 4.6 months), ORR ( $66 \%$ vs $15 \%)$ and CBR ( $67 \%$ vs $40 \%$ ) were observed. The benefits of palbociclib/fulvestrant in PFS compared to fulvestrant/placebo were consistent irrespective of the degree of HR expression, PIK3CA mutation, ET resistance and ethnicity. These findings propose the possibility of re-sensitising endocrine sensitivity in ET-resistant tumours by targeting of CDK4/6. Common toxicities include: neutropenia, leukopenia, fatigue and anaemia were observed in ET/palbociclib arms. These haematological changes should be considered during patient selection for this therapeutic strategy. Endocrine monotherapy had limited efficacy in patients with PD from prior ET, proposing a need for further investigations into the effective use of combination regimens to overcome this problem (Cristofanilli et al. 2016).

\section{ET combinations with PI3K inhibitors (phase III)}

BELLE-2 was a randomised, double-blinded, placebocontrolled phase III trial that investigated the addition of buparlisib to fulvestrant. Overall, promising results were observed; with PFS, ORR and CBR all being improved in the experimental arm. The toxicity profile of the addition of buparlisib seems to be associated with liver function, with increase in alanine aminotransferase (26\% vs $1 \%$ and aspartate aminotransferase (18\% vs $3 \%)$ ). Hence, the use of buparlisib in patients with poor liver function should be cautioned. Interestingly, Baselga et al. reported that buparlisib significantly improved median PFS, ORR and CBR in patients with PIK3CAmutant ctDNA, but the same activity was not observed in patients without the mutation. Furthermore, patients characterised with PIK3-mutated tumours are associated with endocrine-resistant HR+, HER2- ABC (Baselga et al . 2016). This proposes the possibility that the targeting of PI3K pathway may be an area to explore for overcoming endocrine resistance.

\section{ET combinations with HER2 inhibitors (phase III)}

Positive results of adding HER2 inhibitor to ET was shown in the TAnDEM study (anastrozole/trastuzumab) and in a phase III study that investigated letrozole in combination with lapatinib (Johnston et al. 2009, Kaufman et al. 2009, Burstein et al. 2014). PFS and CBR were greatly enhanced, with a doubling of PFS was seen in both studies (Table 3). However, the increase in PFS did not correlate with OS. More AEs were reported in the combination arm in both studies. Moreover, an increase in cardiac events 
(14 vs 2) was observed in anastrozole/trastuzumab when compared to anastrozole alone. Johnston et al. also discussed the problem of ET resistance in HR+, HER $2+$ breast cancer and concluded that the addition of lapatinib did not delay disease progression with letrozole in endocrine-sensitive tumours. In general, the studies concur that addition of HER2 inhibitors to ET in HR+, HER2+ breast cancer can prolong chemoprevention and increase ET efficacy.

CALGB 40302 was a randomised, double-blinded, placebo-controlled phase III study that investigated the fulvestrant/lapatinib combination. Conversely, there was a lack of improvement in clinical outcomes. Though, it was noted that PFS was improved in patients with HER2+ tumours (5.9 vs 3.3 months) as opposed to HER2 - tumours (4.1 vs 3.8 months) when lapatinib was added. However, this study had a small number of HER2+ cases (18\%) with the majority being HER2tumours (81\%). Hence, this could be a limitation of the study that patient recruitment could have been amended to include more HER2+ cases to maximise activity of the HER2 inhibitor. Although the experimental regimen was generally tolerable, there were more AEs and treatment discontinuation caused from the raised toxicity. Overall, CALGB 40302 concluded that lapatinib did not significantly improve clinical benefits when added to fulvestrant (Burstein et al. 2014).

\section{ET combinations with VEGFR inhibitor (phase III)}

From Table 3, the CALGB 40503 (letrozole with bevacizumab) and LEA study (letrozole/fulvestrant with bevacizumab), reported of contrasting findings in PFS. According to the CALGB 40503 study, the addition of bevacizumab to letrozole improved PFS (20.2 vs 15.6 months) when compared to the placebo arm. Moreover, ORR (69\% vs $49 \%$ ) and CBR ( $80 \%$ vs $62 \%$ ) exhibited similar improvements from the addition of bevacizumab. However, the significant improvement in PFS, ORR and CBR did not correlate with OS (47.2 vs 43.9 months) (Dickler et al. 2016). Similar improvements in PFS (19.3 vs 14.4 months), ORR (41\% vs $22 \%$ ) and CBR (77\% vs 66\%) were observed in the LEA study. However, the difference in PFS was not statistically significant: the hazard ratio of the combination arm vs ET alone was $0.83(P=0.126)$ (Martin et al. 2015). Unsurprisingly, bevacizumab combinations were associated with increased AEs; mainly hypertension and proteinuria. The LEA study reported of deaths in the bevacizumab arm that seem to be associated with conditions that may have been worsened from the hypertensive side effects (Martin et al. 2015). As a result, patients with hypertensive conditions should avoid the use of bevacizumab.

One of the limitations of the LEA study was the lack of comparison of letrozole and fulvestrant when in combination with bevacizumab. All the data assimilated were grouped together either as ET/bevacizumab and ET alone. Further sub-groups within ET/bevacizumab to compare letrozole/bevacizumab and fulvestrant/ bevacizumab would have provided more information on optimal application of bevacizumab to ET.

\section{ET combinations with EGFR inhibitor (phase II)}

Marked advantage in PFS was reported when gefitinib was added to anastrozole in comparison to placebo (Table 4) (Valero et al. 2009, Cristofanilli et al. 2010). Improvement in PFS was also observed in the study of tamoxifen in combination with gefitinib. For this trial, patients were split into two groups: stratum 1 (PD after tamoxifen) and 2 (PD during/after AI). PFS was only improved in stratum 1 (10.9 vs 8.8 months), but not in stratum 2 (5.7 vs 7.0 months). The significant improvement of PFS in stratum 1 suggests possible endocrine re-sensitisation when gefitinib was added to an ET (tamoxifen, in this case) that was previously used (Osborne et al. 2011). A sub-analysis of PFS in patients with prior ET therapies (11.2 vs 7.1 months) and ET naïve (20.2 vs 8.4 months) was observed using gefitnib/ anastrozole vs placebo arm (Cristofanilli et al. 2010). These findings suggest a potential role of overcoming ET resistance from using gefitinib. On the other hand, Tryfonidis et al. argued that the toxicity profile (mainly skin and gastrointestinal related) of gefitinib resulted in premature therapy interruption in 33\% of patients. Additionally, the PFS rate at 1 year was only $35 \%$ for combination arm and 32\% for placebo arm (Tryfonidis et al. 2016). Hence, the use of gefitinib was not supported in a risk/benefit point of view. Carlson et al. echoed similar opinion in further trials of combinations of gefitnib with anastrozole/fulvestrant, despite modest findings in anti-tumour activities (Carlson et al. 2012). Overall, PFS comparison seemed similar (5.3 vs 5.2 months in anastrozole and fulvestrant arms respectively) but in patients who had prior chemotherapy, a significant deterioration in PFS was seen in the fulvestrant/gefitinib arm (2.6 months) (Tryfonidis et al. 2016). Although it was unexplained why these changed were observed, it can be inferred that prior treatment can have an impact on future treatments. 


\section{ET combinations with TKI (phase II)}

The general consensus toward TKI/ET combinations seems negative. Johnston et al. reported a 3 arms trial of anastrozole $(1 \mathrm{mg} /$ day $)$ in combination with AZD8931 at $20 \mathrm{mg}$ (twice daily), $40 \mathrm{mg}$ (twice daily) or placebo. Although PFS (13.8 vs 14.9 vs 10.9 months) was increased, it was statistically insignificant (Table 4) (Johnston et al. 2016). This therapeutic strategy does not seem to enhance ET responsiveness and was generally associated with a greater toxicity profile when compared to ET alone. Wright et al. reported that the addition of dasatinib to fulvestrant did not improve PFS (6.0 months vs 5.3 months), CBR and OS. In fact, CBR $(28.0 \%$ vs $32.7 \%$ ) and OS (17.0 vs 21.7 months) seemed to worsen with dasatinib/fulvestrant when compared to placebo (Wright et al. 2011). This may suggest that a worse safety profile and patient tolerability could potentially influence the patient's QoL and ultimately OS. Finally, in the fulvestrant/dovitinib study, an improvement in PFS (10.9 vs 5.5 months) was observed, although this was only limited to patients with FGF pathway-amplified breast cancer in fulvestrant/dovitinib vs placebo arm respectively. Contrastingly, patients without FGF pathway amplification gained no effect from the addition of dovitinib (5.5 vs 5.5 months), other than the increased toxicity associated in combination therapy (Musolino et al. 2017). This discovery highlights the importance of patient selection by identifying cancer biology to maximise treatment prognosis.

\section{Sequential therapy search}

From Fig. 3, an initial detection of 901 articles. A final total of 0 studies were identified, after removal of duplicates, title and abstract screening according to the inclusion and exclusion criteria stated in methods. Therefore, the search for relevant literature in the sequential application of ET and TA was unsuccessful.

\section{Discussion}

This review aimed to explore options for the optimisation of ET with TA by methods of combination therapy or sequential therapy. From assimilating relevant studies, it was clear that combination therapy is investigated more thoroughly than sequential therapy. The identification of benefits and limitations in both combination and sequential therapy was not met due to the absence of literature available in sequential therapy. The result of
0 articles warrants the need of future investigation in this area.

It was hypothesised that combination therapy would be the better option in optimising ET. Most combinations of ET and TA have yielded extremely promising results, notably in enhancing treatment efficacy (PFS, ORR and CBR). The classes of TA reviewed in this systematic review included: mTOR inhibitors, EGFR inhibitors, TKI, CDK4/6 inhibitors, VEGFR inhibitors, PI3K inhibitor and HER2 inhibitors. Most treatment combinations were effective in treating patients with $\mathrm{HR}+$, HER2- ABC/MBC. Evidently, the best combination arms included CDK4/6 inhibitor, PI3K inhibitor and mTOR inhibitors in treating this population. These combinations seem to optimise ET by producing significant improvements in PFS, CBR and ORR, regardless of patients' treatment history and overcoming endocrine resistant. The additional benefits from combination therapy were associated with an increase in toxicity. This was a common trend in all included studies. Consequently, combination therapy may prove difficult in patients whom do not tolerate these regimens, for instance, in the elderly population.

All studies documented the toxicity profile of the combination against the comparison arm. However, it was unknown how these toxicities may have impacted the patient being treated. Most studies had stated that one of the main reasons for patient discontinuation was related to treatment toxicity. Data in these areas should identify treatment tolerability, patients' QoL and financial feasibility for sustainable treatment. Therefore, clinicians will be provided with a better understanding on the ideal application of ET and TA.

Throughout the review, it was evident that some combinations (TKI, EGFR and VEGFR) failed to produce any benefits over ET alone. Differences in study design seemed to be the most likely explanation for contrasting findings in RCTs with similar experimental arms. Most RCTs used methods such as: doubleblinding, placebo-controlled and 2-arm trial. Although some RCTs deviated from this and employed an openlabel approach and the absent of placebo. Hence, those RCTs may be of lower power than those that used the double-blinding and placebo-control methods to minimise chances of bias.

\section{Patient selection}

It was implied that the importance of patient selection seemed to influence treatment prognosis. From assimilating relevant study findings, this review suggests 

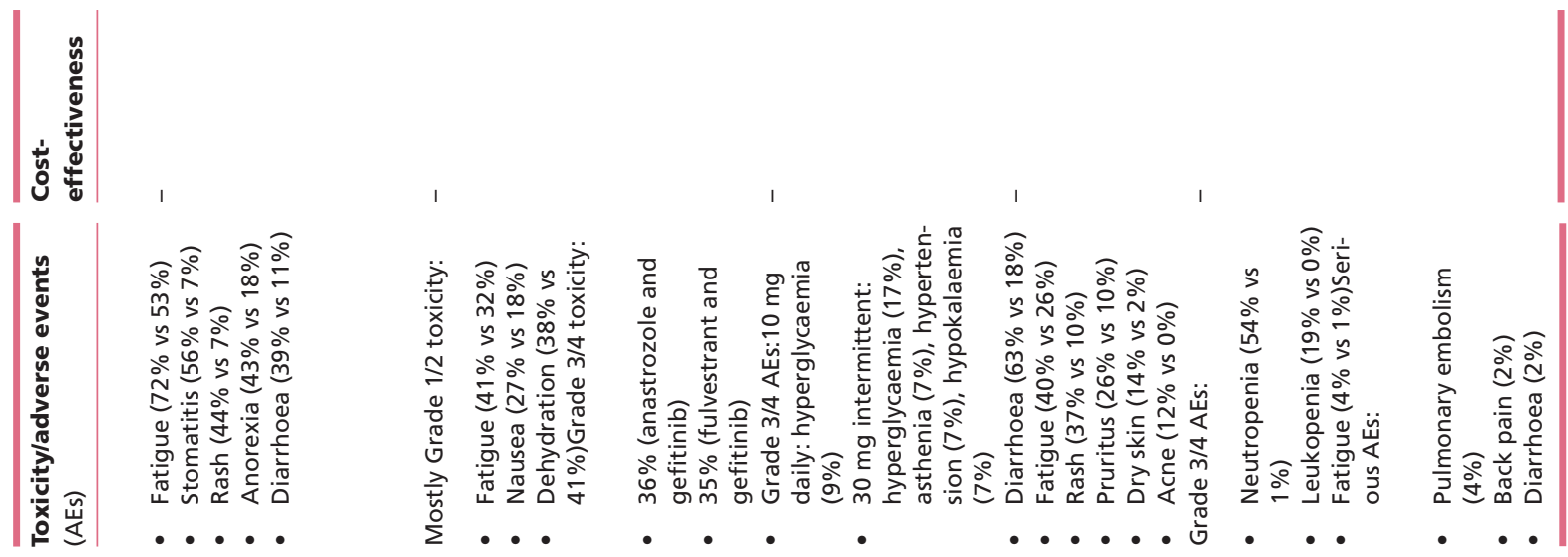

$\vec{a}$
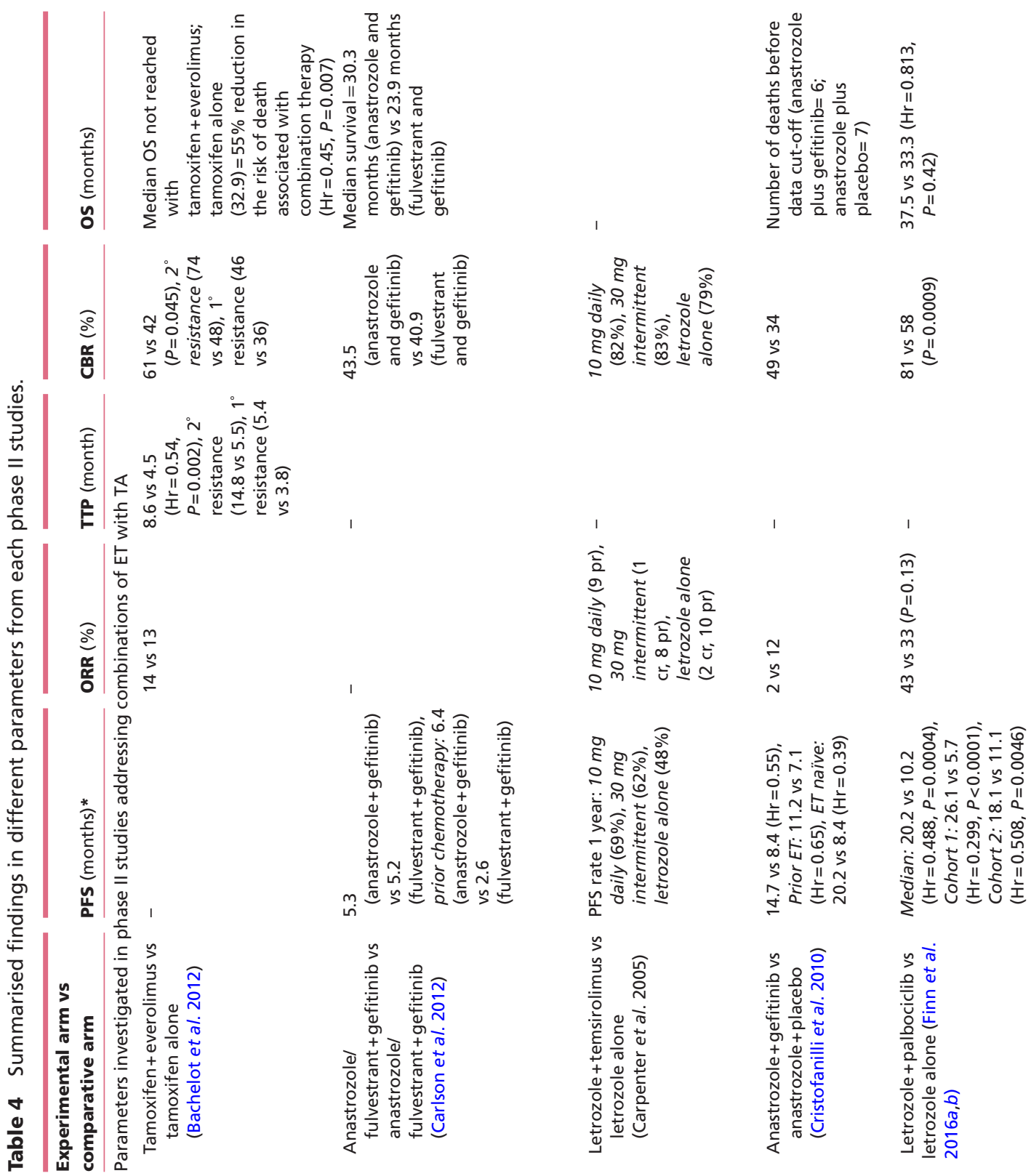

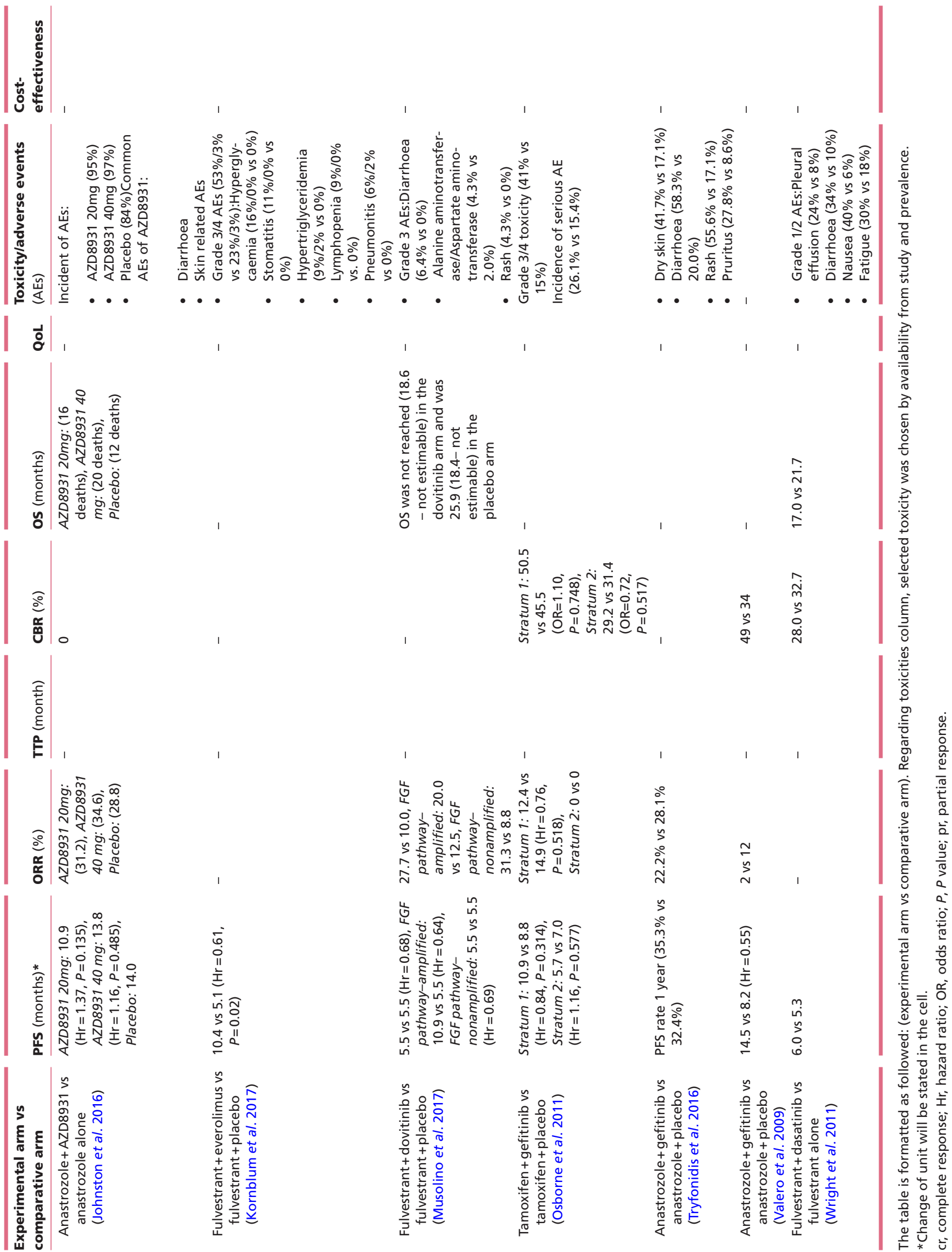

ஃ̊
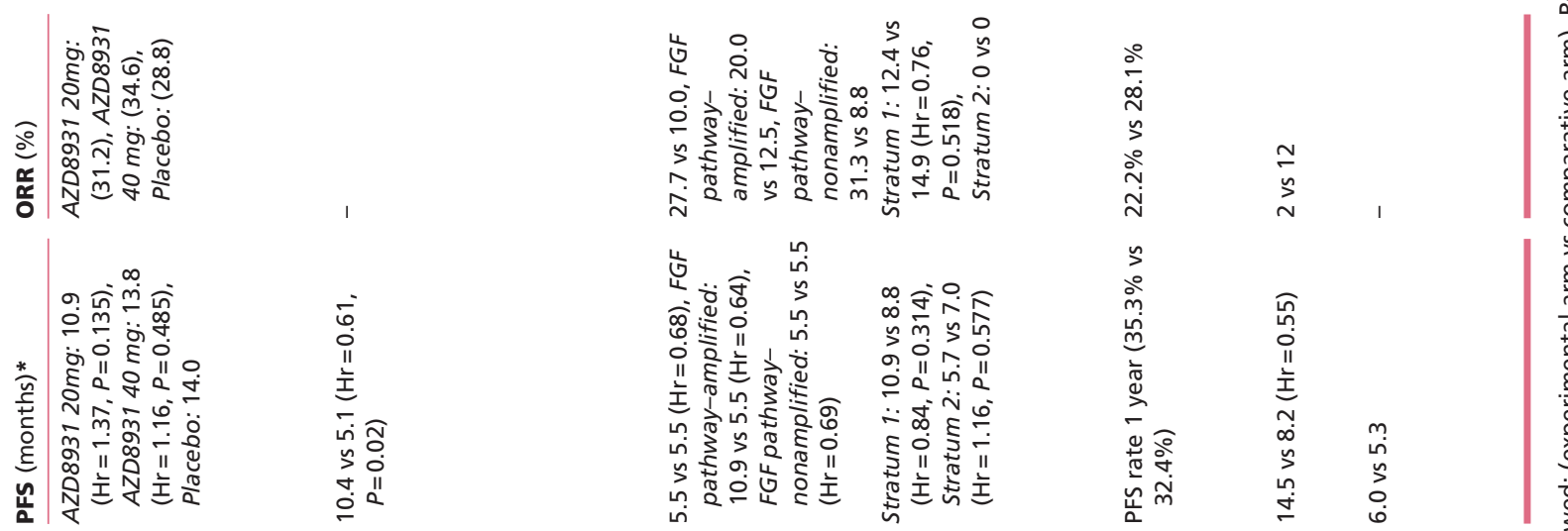

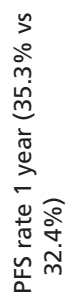
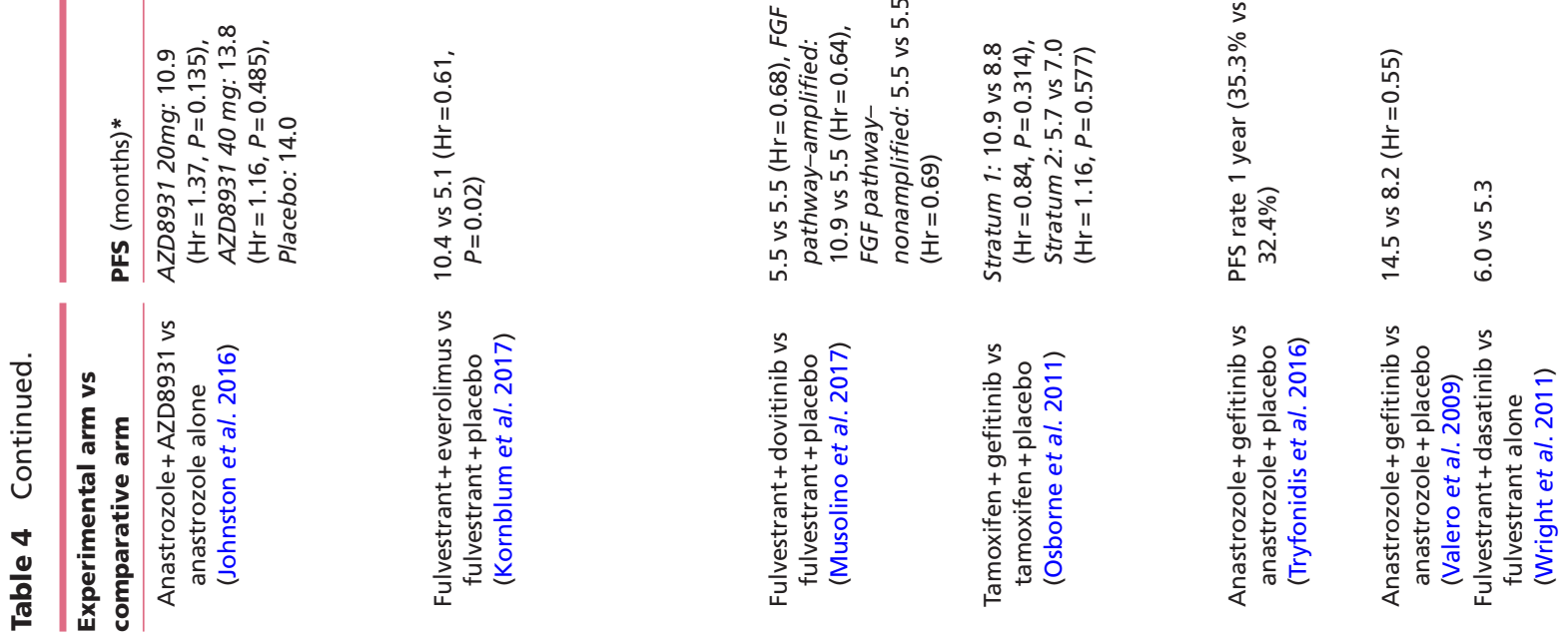
that patient selection can be categorised into three main areas: patient characteristics, cancer biology and pharmacology.

\section{Patient characteristics}

Patient characteristics such as age have shown to influence drug efficacy. In the HORIZON study, temsirolimus produced PFS benefits in younger patients as opposed to older patients (Wolff et al. 2013). Thus, the use of SERMs and SERDs in combination to temsirolimus may exhibit greater benefit in selected younger patients than using AIs, which are restricted to the postmenopausal population. However, it should be reminded that not all postmenopausal patients are of the older population. Younger patients can obtain the postmenopausal status via oophorectomy or the use of a luteinising hormonereleasing hormone agonist. Another aspect to consider in older patients would be treatment tolerability. From the LEA study, details of patients' deaths were reported in the bevacizumab arm $(n=8)$ (Martin et al. 2015). Some deaths were associated with conditions that may have been exacerbated from the hypertensive side effects. Further inspection, revealed that the patient age ranged from 53 to 82 years and 5 out of 8 patients had hypertension as baseline co-morbidity (Martin et al. 2015). Therefore, specific co-morbidities in individual patients should be considered when selecting regimens. As evidently different classes of TA are associated with specific toxicities: palbociclib (neutropenia), bevacizumab (hypertension), trastuzumab (cardiac events) and EGFR inhibitors (skin and gastrointestinal).

\section{Studies addressing sequential ET with TA}

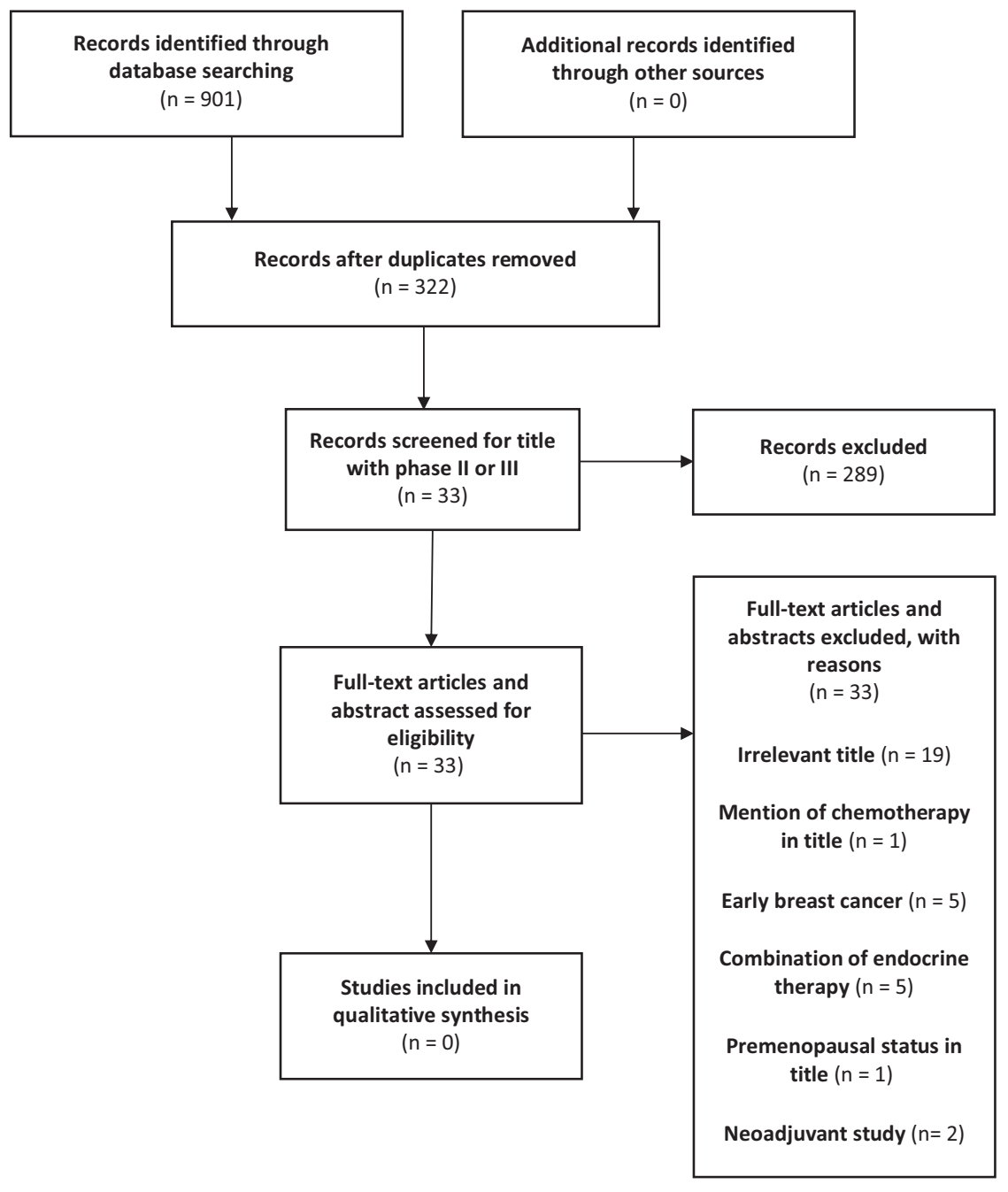

Figure 3

A flow diagram displaying the study selection process that addressed for sequential use of ET with targeted agents. Adapted, under the terms of the Creative Commons Attribution License, from Moher D, Liberati A, Tetzlaff J, Altman DG, The PRISMA Group (2009) Preferred Reporting Items for Systematic Reviews and Meta-Analyses: The PRISMA Statement. PLoS Medicine 6(7): e1000097. 


\section{Cancer biology}

The identification of specific targets can broaden the options for therapeutic strategies. For instance, the use of dovitinib (TKI that inhibits FGF pathways) in combination with fulvestrant was shown to significantly improve PFS in patients with FGF pathway-amplified breast cancer (10.9 vs 5.5 months) when compared to the placebo arm. However, patients without FGF pathway amplification did not benefit from the dovitinib/fulvestrant combination (5.5 vs 5.5 months) (Musolino et al. 2017). Burstein et al. also reported greater improvement in PFS and ORR, when the HER2 inhibitor lapatinib was added to fulvestrant in patients with HER2+ status than in those with HER2- (Burstein et al. 2014). These findings support the importance of patient selection, by identifying cancer biology to maximise treatment success.

\section{Pharmacology}

Pharmacology was another factor that should be considered during patient selection for suitable therapeutic strategy. It was clear from the findings in this systematic review, that prior therapy can influence treatment prognosis. This was evident in studies of ET/ EGFR combinations, whereby prior ET or chemotherapy had caused dramatic changes in treatment outcome. In the phase II study that investigated the anastrozole/ gefitinib combination, Cristofanilli et al. reported an exploratory post hoc subset analysis of patients with endocrine naïve and prior ET. An all-round improvement in PFS was observed in both subsets. But, the data seem to suggest superior benefits in PFS for patients with endocrine naïve (20.2 months) in contrast to patients who had prior ET (11.2 months) (Cristofanilli et al. 2016). From these findings, it was confirmed that endocrine monotherapy had limited efficacy in patients with PD from prior ET, proposing a need for further investigations into the effective selection of combination regimens to overcome this problem. Furthermore, this proposes that the use of combination therapy in a first-line setting may benefit those with naïve treatment. Although some combinations (CDK4/6, PI3K, EGFR and mTOR) have shown activity to overcome ET resistance in patients with prior ET exposure. Yet, it was unspecified if the number of prior therapies may further diminish the outcome in combination therapy. Hence, this may be another area to be for future investigations.

\section{Overcoming resistance}

One of the criteria for optimising ET in this review was to overcome ET resistance. This question was met in findings from phase III PALOMA-3 and BELLE-2 studies suggesting that targeting CDK4/6 and PI3K hold the most promise. This was supported by in vitro evidence suggesting cancer cells that have developed ET resistance remain dependent on cyclin D1 and CDK4 for proliferation. Similarly, preclinical evidence has identified a potential cause of endocrine resistance via cross-talk between ER and PI3K pathways (Milani et al. 2014). Additional findings from phase II ET combinations with gefitinib and everolimus suggested signs of delaying ET resistance or re-sensitising tumours with ET resistance promise (Bachelot et al. 2012, Tryfonidis et al. 2016). This prompts further research into overcoming ET resistance by targeting these pathways.

\section{Sequential application}

There was evidently a lack of knowledge about the sequential application of ET and TA. This review has identified areas that combination therapy has failed to impress, and a new approach in optimal application of specific target agents was needed. For instance, the activity of gefitinib with ET has suggested effects of delaying ET resistance. But in a combination setting, the regimen seemed to only increase toxicity while retaining similar efficacy seen in endocrine monotherapy (Tryfonidis et al. 2016). Hence, the sequential application of these agents could be a feasible alternative. A predicted decrease in toxicity would provide a more tolerable profile for patients. This will be important for management of the elderly population where tolerability may be an issue. Classes of TA such as TKIs, VEGFR inhibitors and HER2 inhibitors when in combination created unfavourable tolerability in patients. Therefore, those classes of agents may benefit from this sequential approach.

\section{Limitations}

The term 'targeted agents' was narrowly defined to fit the feasibility of generating this systematic review. Several agents were excluded from this review included: proteasome inhibitors and farnesyltransferase inhibitors. Moreover, combination therapy was strictly defined to only include 2 agent combinations and excluding studies that have explored the feasibility of more than 
2 agent combinations such as triple combinations. Thus, this review does not reflect the true potential depth of combination therapy and diversity of TA available for optimising ET.

The method in selecting papers was rigorously determined by the presence of specific keywords. Studies that were excluded solely based on title alone, may have contained relevant information in the abstract or within the full text. Thus, there was the possibility that relevant studies were missed.

Furthermore, many trial status were 'on-going' or 'results pending', this resulted in a narrow range of agents being incorporated into this review. This was especially evident in the attempt of including novel agents that targeted the PI3K pathway. Consequently, the protocol was amended to allow inclusion of abstracts to generate a wider pool of agents and subsequent findings. However, limited information was provided in the abstracts when compared to full text. This was evident during analysis of study design and results.

\section{Conclusion}

Combination of ET and TA has proven to be effective at improving treatment efficacy over monotherapy in postmenopausal patients with $\mathrm{HR}+\mathrm{ABC} / \mathrm{MBC}$. However, not all combinations are adding benefit to ET and some are only increasing the toxicity profile. Indisputably, tolerability of toxicity in combination therapy of the elderly population possesses an issue in patient management. As a result, this may be an opportunity for sequential therapy of ET and TA to be explored in this specific population.

\section{Declaration of interest}

Thomas Ho Lai Yau has declared no conflict of interest. Kwok-Leung Cheung: research funding - AstraZeneca; consulting or advisory role Genomic Health and travel, accommodation, expenses - Genomic Health.

\section{Funding}

This research did not receive any specific grant from any funding agency in the public, commercial or not-for-profit sector.

\section{References}

Bachelot T, Bourgier C, Cropet C, Ray-Coquard I, Ferrero JM, Freyer G, Abadie-Lacourtoisie S, Eymard JC, Debled M, Spaeth D, et al. 2012
Randomized phase II trial of everolimus in combination with tamoxifen in patients with hormone receptor-positive, human epidermal growth factor receptor 2-negative metastatic breast cancer with prior exposure to aromatase inhibitors: a GINECO Study. Journal of Clinical Oncology 30 2718-2724. (https://doi.org/10.1200/ JCO.2011.39.0708)

Baselga J, Campone M, Piccart M, Burris IHA, Rugo HS, Sahmoud T, Noguchi S, Gnant M, Pritchard KI, Lebrun F, et al. 2012 Everolimus in postmenopausal hormone-receptor-positive advanced breast cancer. New England Journal of Medicine 366 520-529. (https://doi. org/10.1056/NEJMoa1109653)

Baselga J, Im SA, Iwata H, Clemons M, Ito Y, Awada A, Chia S, JagielloGruszfeld A, Pistilli B, Tseng LM, et al. 2016 PIK3CA status in circulating tumor DNA (ctDNA) predicts efficacy of buparlisib (BUP) plus fulvestrant (FULV) in postmenopausal women with endocrineresistant $\mathrm{HR}+$ /HER2-advanced breast cancer (BC): first results from the randomized, phase III BELLE-2 trial. Cancer Research 76 (4 Suppl) abstract S6-01. (https://doi.org/10.1158/1538-7445. SABCS15-S6-01)

Burris H, Gnant M, Hortobagyi G, Hart L, Yardley DA, Eakle J, Provencher L, Brechenmacher T, Saletan S, Taran T, et al. $2013 a$ Characterization of response to everolimus (EVE) in BOLERO-2: a phase 3 trial of EVE plus exemestane (EXE) in postmenopausal women with HR+, HER2-advanced breast cancer. Cancer Research 73 (24 Suppl) abstract P2-16-17. (https://doi.org/10.1158/0008-5472. SABCS13-P2-16-17)

Burris HA, Lebrun F, Rugo HS, Beck JT, Piccart M, Neven P, Baselga J, Petrakova K, Hortobagyi GN, Komorowski A, et al. 2013b Healthrelated quality of life of patients with advanced breast cancer treated with everolimus plus exemestane versus placebo plus exemestane in the phase 3, randomized, controlled, BOLERO-2 trial. Cancer 119 1908-1915. (https://doi.org/10.1002/cncr.28010)

Burstein HJ, Cirrincione CT, Barry WT, Chew HK, Tolaney SM, Lake DE Ma C, Blackwell KL, Winer EP \& Hudis CA 2014 Endocrine therapy with or without inhibition of epidermal growth factor receptor and human epidermal growth factor receptor 2: a randomized, doubleblind, placebo-controlled phase III trial of fulvestrant with or without lapatinib for postmenopausal women with hormone receptor-positive advanced breast cancer - CALGB 40302 (alliance). Journal of Clinical Oncology 32 3959-3966. (https://doi.org/10.1200/ JCO.2014.56.7941)

Carlson RW, O’Eill A, Vidaurre T, Gomez HL, Badve SS \& Sledge GW 2012 A randomized trial of combination anastrozole plus gefitinib and of combination fulvestrant plus gefitinib in the treatment of postmenopausal women with hormone receptor positive metastatic breast cancer. Breast Cancer Research and Treatment 133 1049-1056. (https://doi.org/10.1007/s10549-012-1997-5)

Carpenter J, Roché H, Campone M, Colomer R, Jagiello-Gruszfeld A, Moore L, D'Amore M, Kong S \& Boni J 2005 Randomized 3-arm, phase 2 study of temsirolimus (CCI-779) in combination with letrozole in postmenopausal women with locally advanced or metastatic breast cancer. Journal of Clinical Oncology 23 (16_Suppl) 564. (https://doi.org/10.1200/jco.2005.23.16_suppl.564)

Cheung KL 2007 Endocrine therapy for breast cancer: an overview. Breast 16 327-343. (https://doi.org/10.1016/j.breast.2007.03.004)

Cristofanilli M, Valero V, Mangalik A, Royce M, Rabinowitz I, Arena FP, Kroener JF, Curcio E, Watkins C, Bacus S, et al. 2010 Phase II, randomized trial to compare anastrozole combined with gefitinib or placebo in postmenopausal women with hormone receptor-positive metastatic breast cancer. Clinical Cancer Research 16 1904-1914. (https://doi.org/10.1158/1078-0432.CCR-09-2282)

Cristofanilli M, Turner NC, Bondarenko I, Ro J, Im S-A, Masuda N, Colleoni M, DeMichele A, Loi S, Verma S, et al. 2016 Fulvestrant plus palbociclib versus fulvestrant plus placebo for treatment of hormone-receptor-positive, HER2-negative metastatic breast cancer
(2) 2018 Society for Endocrinology Published by Bioscientifica Ltd. Printed in Great Britain 
that progressed on previous endocrine therapy (PALOMA-3): final analysis of the multicentre, double-blind, phase 3 randomised controlled trial. Lancet Oncology 17 425-439. (https://doi org/10.1016/S1470-2045(15)00613-0)

Dickler MN, Barry WT, Cirrincione CT, Ellis MJ, Moynahan ME, Innocenti F, Hurria A, Rugo HS, Lake DE, Hahn O, et al. 2016 Phase III trial evaluating letrozole as first-line endocrine therapy with or without bevacizumab for the treatment of postmenopausal women with hormone receptor-positive advanced-stage breast cancer: CALGB 40503 (Alliance). Journal of Clinical Oncology 34 2602-2609. (https://doi.org/10.1200/JCO.2015.66.1595)

Dixon JM 2014 Endocrine resistance in breast cancer. New Journal of Science 2014 27. (https://doi.org/10.1155/2014/390618)

Finn RS, Martin M, Rugo HS, Jones S, Im SA, Gelmon K, Harbeck N, Lipatov ON, Walshe JM, Moulder S, et al. 2016a Palbociclib and letrozole in advanced breast cancer. New England Journal of Medicine 375 1925-1936. (https://doi.org/10.1056/NEJMoa1607303)

Finn RS, Martin M, Rugo HS, Jones SE, Im SA, Gelmon KA, Harbeck N, Lipatov ON, Walshe JM, Moulder SL, et al. $2016 b$ Paloma-2: primary results from a phase III trial of palbociclib (P) with letrozole $(\mathrm{L})$ compared with letrozole alone in postmenopausal women with ER+/HER2-advanced breast cancer (ABC). Journal of Clinical Oncology 34 (15_Suppl) 507. (https://doi.org/10.1200/ JCO.2016.34.15_suppl.507)

Hammond MEH, Hayes DF, Dowsett M, Allred DC, Hagerty KL, Badve S, Fitzgibbons PL, Francis G, Goldstein NS, Hayes M, et al. 2010 American Society of Clinical Oncology/College of American Pathologists Guideline recommendations for immunohistochemical testing of estrogen and progesterone receptors in breast cancer (unabridged version). Archives of Pathology and Laboratory Medicine 134 e48-e72. (https://doi. org/10.1043/1543-2165-134.7.e48)

Jeselsohn R, Buchwalter G, De Angelis C, Brown M \& Schiff R 2015 ESR1 mutations as a mechanism for acquired endocrine resistance in breast cancer. Nature Reviews: Clinical Oncology 12 573-583. (https:// doi.org/10.1038/nrclinonc.2015.117)

Johnston S, Pippen J Jr, Pivot X, Lichinitser M, Sadeghi S, Dieras V, Gomez HL, Romieu G, Manikhas A, Kennedy MJ, et al. 2009 Lapatinib combined with letrozole versus letrozole and placebo as first-line therapy for postmenopausal hormone receptor - positive metastatic breast cancer. Journal of Clinical Oncology 27 5538-5546. (https://doi.org/10.1200/JCO.2009.23.3734)

Johnston S, Basik M, Hegg R, Lausoontornsiri W, Grzeda L, Clemons M, Dreosti L, Mann H, Stuart M \& Cristofanilli M 2016 Inhibition of EGFR, HER2, and HER3 signaling with AZD8931 in combination with anastrozole as an anticancer approach: phase II randomized study in women with endocrine-therapy-naïve advanced breast cancer. Breast Cancer Research and Treatment 160 91-99. (https://doi. org/10.1007/s10549-016-3979-5)

Kaufman B, Mackey JR, Clemens MR, Bapsy PP, Vaid A, Wardley A, Tjulandin S, Jahn M, Lehle M, Feyereislova A, et al. 2009 Trastuzumab plus anastrozole versus anastrozole alone for the treatment of postmenopausal women with human epidermal growth factor receptor 2-positive, hormone receptor-positive metastatic breast cancer: results from the randomized phase III TAnDEM study. Journal of Clinical Oncology 27 5529-5537. (https://doi.org/10.1200/ JCO.2008.20.6847)

Kornblum NS, Manola J, Klein P, Ramaswamy B, Brufsky A, Stella PJ, Burnette B, Telli M, Makower DF, Leach J, et al. 2017 Abstract S1-02: PrECOG 0102: A randomized, double-blind, phase II trial of fulvestrant plus everolimus or placebo in post-menopausal women with hormone receptor (HR)-positive, HER2-negative metastatic breast cancer (MBC) resistant to aromatase inhibitor (AI) therapy. Cancer Research 77 (4 Suppl) abstract S1-02. (https://doi. org/10.1158/1538-7445.SABCS16-S1-02)
Martin M, Loibl S, Von Minckwitz G, Morales S, Martinez N, Guerrero A, Anton A, Aktas B, Schoenegg W, Munoz M, et al. 2015 Phase III trial evaluating the addition of bevacizumab to endocrine therapy as firstline treatment for advanced breast cancer: the Letrozole/Fulvestrant and Avastin (LEA) study. Journal of Clinical Oncology 33 1045-1052. (https://doi.org/10.1200/JCO.2014.57.2388)

Milani A, Geuna E, Mittica G \& Valabrega G 2014 Overcoming endocrine resistance in metastatic breast cancer: current evidence and future directions. World Journal of Clinical Oncology 5 990-1001. (https://doi.org/10.5306/wjco.v5.i5.990)

Moher D, Liberati A, Tetzlaff J, Altman DG \& The PG 2009 Preferred reporting items for systematic reviews and meta-analyses: the PRISMA statement. PLOS Medicine 6 e1000097. (https://doi. org/10.1371/journal.pmed.1000097)

Musolino A, Campone M, Neven P, Denduluri N, Barrios CH, Cortes J, Blackwell K, Soliman H, Kahan Z, Bonnefoi H, et al. 2017 Phase II, randomized, placebo-controlled study of dovitinib in combination with fulvestrant in postmenopausal patients with HR+, HER2(-) breast cancer that had progressed during or after prior endocrine therapy. Breast Cancer Research 19 18. (https://doi.org/10.1186/ s13058-017-0807-8)

Osborne CK, Neven P, Dirix LY, Mackey JR, Robert J, Underhill C, Schiff R, Gutierrez C, Migliaccio I, Anagnostou VK, et al. 2011 Gefitinib or placebo in combination with tamoxifen in patients with hormone receptor-positive metastatic breast cancer: a randomized phase II study. Clinical Cancer Research 17 1147. (https://doi. org/10.1158/1078-0432.CCR-10-1869)

Piccart M, Baselga J, Noguchi S, Burris H, Gnant M, Hortobagyi G, Mukhopadhyay P, Taran T, Sahmoud T \& Rugo H 2012 Final progression-free survival analysis of BOLERO-2: a phase III trial of everolimus for postmenopausal women with advanced breast cancer. Cancer Research 72 (24 Suppl) abstract P6-04-02. (https://doi. org/10.1158/0008-5472.SABCS12-P6-04-02)

Pietras RJ 2006 Biologic basis of sequential and combination therapies for hormone-responsive breast cancer. Oncologist 11 704-717. (https://doi.org/10.1634/theoncologist.11-7-704)

Reinert T \& Barrios CH 2015 Optimal management of hormone receptor positive metastatic breast cancer in 2016. Therapeutic Advances in Medical Oncology 7 304-320. (https://doi. org/10.1177/1758834015608993)

Robertson JFR, Bondarenko IM, Trishkina E, Dvorkin M, Panasci L, Manikhas A, Shparyk Y, Cardona-Huerta S, Cheung K-L, PhilcoSalas MJ, et al. 2016 Fulvestrant $500 \mathrm{mg}$ versus anastrozole $1 \mathrm{mg}$ for hormone receptor-positive advanced breast cancer (FALCON): an international, randomised, double-blind, phase 3 trial. Lancet 388 2997-3005. (https://doi.org/10.1016/S01406736(16)32389-3)

Roche PC \& Ingle JN 1999 Increased HER2 with U.S. food and drug administration-approved antibody. Journal of Clinical Oncology 17 434-434. (https://doi.org/10.1200/JCO.1999.17.1.434)

Tryfonidis K, Basaran G, Bogaerts J, Debled M, Dirix L, Thery J, TjanHeijnen V, Weyngaert D, Cufer T, Piccart M, et al. 2016 A European Organisation for Research and Treatment of Cancer randomized, double-blind, placebo-controlled, multicentre phase II trial of anastrozole in combination with gefitinib or placebo in hormone receptor-positive advanced breast cancer (NCT00066378). European Journal of Cancer 53 144-154. (https://doi.org/10.1016/j. ejca.2015.10.012)

Valero V, Bacus S, Mangalik A, Rabinowitz I, Arena F, Kroener J, Curcio E, Watkins C, Magill P \& Cristofanilli M 2009 Molecular marker correlates of clinical outcome in a phase II study of gefitinib or placebo in combination with anastrozole in postmenopausal women with hormone receptor-positive metastatic breast cancer. Cancer Research 69 (2 Suppl) abstract 3131. (https://doi. org/10.1158/0008-5472.SABCS-3131)
(2) 2018 Society for Endocrinology Published by Bioscientifica Ltd. Printed in Great Britain 
Wolff AC, Lazar AA, Bondarenko I, Garin AM, Brincat S, Chow L, Sun Y, Neskovic-Konstantinovic Z, Guimaraes RC, Fumoleau P, et al. 2013 Randomized phase III placebo-controlled trial of letrozole plus oral temsirolimus as first-line endocrine therapy in postmenopausal women with locally advanced or metastatic breast cancer. Journal of Clinical Oncology 31 195-202. (https://doi.org/10.1200/ JCO.2011.38.3331)

Wright GL, Blum J, Krekow LK, McIntyre KJ, Wilks ST, Rabe AC, Vukelja SJ, Andersen JC, Wang Y, Asmar L, et al. 2011 Randomized phase II trial of fulvestrant with or without dasatinib in postmenopausal patients with hormone receptor-positive metastatic breast cancer previously treated with an aromatase inhibitor. Cancer Research 71 (24 Suppl) abstract PD01-01. (https://doi. org/10.1158/0008-5472.SABCS11-PD01-01)

Yardley DA, Noguchi S, Pritchard KI, Burris HA, Baselga J, Gnant M, Hortobagyi GN, Campone M, Pistilli B, Piccart M, et al. 2013 Everolimus plus exemestane in postmenopausal patients with $\mathrm{HR}+$ breast cancer: BOLERO-2 final progression-free survival analysis. Advances in Therapy 30 870-884. (https://doi.org/10.1007/s12325013-0060-1)

Received in final form 28 March 2018

Accepted 19 April 2018

Accepted Preprint published online 19 April 2018
(C) 2018 Society for Endocrinology Published by Bioscientifica Ltd. 